\title{
Extreme Variability in a Broad Absorption Line Quasar
}

\author{
Daniel Stern ${ }^{1}$, Matthew J. Graham ${ }^{2}$, Nahum Arav $^{3}$, S. G. Djorgovski ${ }^{2}$, Carter Chamberlain ${ }^{3}$, Aaron J. Barth ${ }^{4}$, Ciro Donalek ${ }^{2}$, \\ Andrew J. Drake ${ }^{2}$, Eilat Glikman ${ }^{5}$, Hyunsung D. Jun ${ }^{1}$, Ashish A. Mahabal ${ }^{2}$, and Charles. C. Steidel ${ }^{2}$ \\ ${ }^{1}$ Jet Propulsion Laboratory, California Institute of Technology, 4800 Oak Grove Drive, Mail Stop 169-221, Pasadena, CA 91109, USA; daniel.k.stern@jpl.nasa.gov \\ ${ }^{2}$ California Institute of Technology, 1200 E. California Boulevard, Pasadena, CA 91125, USA \\ ${ }^{3}$ Department of Physics, Virginia Tech, Blacksburg, VA 24061, USA \\ ${ }^{4}$ Department of Physics and Astronomy, 4129 Frederick Reines Hall, University of California, Irvine, CA 92697, USA \\ ${ }^{5}$ Department of Physics, Middlebury College, Middlebury, VT 05753, USA \\ Received 2017 January 13; revised 2017 February 14; accepted 2017 February 15; published 2017 April 21
}

\begin{abstract}
CRTS J084133.15 +200525.8 is an optically bright quasar at $z=2.345$ that has shown extreme spectral variability over the past decade. Photometrically, the source had a visual magnitude of $V \sim 17.3$ between 2002 and 2008 . Then, over the following five years, the source slowly brightened by approximately one magnitude, to $V \sim 16.2$. Only $\sim 1$ in 10,000 quasars show such extreme variability, as quantified by the extreme parameters derived for this quasar assuming a damped random walk model. A combination of archival and newly acquired spectra reveal the source to be an iron low-ionization broad absorption line quasar with extreme changes in its absorption spectrum. Some absorption features completely disappear over the 9 years of optical spectra, while other features remain essentially unchanged. We report the first definitive redshift for this source, based on the detection of broad $\mathrm{H} \alpha$ in a Keck/MOSFIRE spectrum. Absorption systems separated by several $1000 \mathrm{~km} \mathrm{~s}^{-1}$ in velocity show coordinated weakening in the depths of their troughs as the continuum flux increases. We interpret the broad absorption line variability to be due to changes in photoionization, rather than due to motion of material along our line of sight. This source highlights one sort of rare transition object that astronomy will now be finding through dedicated timedomain surveys.
\end{abstract}

Key words: galaxies: active - quasars: individual (CRTS J084133.15+200525.8)

\section{Introduction}

For galaxies hosting active galactic nuclei (AGNs), timedomain surveys have long proven to be fertile avenues of research. Indeed, optical continuum variability was recognized as a common feature of quasars shortly after their initial discovery (Matthews \& Sandage 1963) and has since been exploited for purposes ranging from identifying quasars (e.g., van den Bergh et al. 1973), to determining black hole masses through reverberation mapping (e.g., Blandford \& McKee 1982; Bentz et al. 2009), to studying the inner circumnuclear environment (e.g., Risaliti et al. 2002). Recent efforts using wide-area, time-domain surveys have vastly extended this avenue of research by exploring the optical variability of extremely large samples of quasars, numbering in the tens to hundreds of thousands (e.g., Macleod et al. 2012; Graham et al. 2014). Besides determining the light curve properties of typical quasars, such work has identified interesting new phenomenology such as candidate periodic light curves suggestive of sub-parsec binary super-massive black hole systems (e.g., D’Orazio et al. 2015a, 2015b; Graham et al. 2015a, 2015b; Jun et al. 2015b; Liu et al. 2015), AGN undergoing major flaring suggestive of microlensing or explosive activity in the accretion disk such as superluminous supernovae, mergers, or tidal disruption events (e.g., Drake et al. 2011; Lawrence et al. 2016; M. J. Graham et al. 2017, in preparation), and changing look AGN with the abrupt appearance or disappearance of broad emission lines (e.g., LaMassa et al. 2015; Gezari et al. 2017).

One topic where quasar variability has received particular attention has been the temporal characteristics of broad absorption line (BAL) quasars. Specifically, over the past few years, several teams have reported on multi-epoch spectroscopic observations of
BAL quasars (e.g., Barlow et al. 1992; Lundgren et al. 2007; Gibson et al. 2008, 2010; Capellupo et al. 2011, 2012, 2013; Filiz et al. 2012, 2013, 2014; Vivek et al. 2012; He et al. 2014, 2015; Joshi et al. 2014; Wildy et al. 2014, 2015; Grier et al. 2015; Zhang et al. 2015). While variability in BAL trough strengths is relatively common, large $(>50 \%)$ changes in the absorption equivalent width is quite rare (e.g., Hall et al. 2011). A primary question in BAL variability studies has been whether observed changes in BAL trough strengths are primarily due to changes in the ionization state of the outflowing wind (e.g., Wang et al. 2015), or whether they are due to high column density BAL clouds moving through our line of sight (e.g., McGraw et al. 2015).

For example, Filiz et al. (2013) present a detailed analysis of $\approx 650$ BAL troughs identified in 291 quasars observed by the Sloan Digital Sky Survey (SDSS), sampling rest-frame timescales between 1 and 3.7 years. They estimate that the average lifetime of a BAL trough is a few thousand years, and that the emergence/disappearance of BAL features are extremes of general BAL variability. Filiz et al. (2013) also report coordinated BAL variability across multiple troughs at different velocities. They argue that changes in the opacity of the shielding gas producing changes in the ionizing radiation incident on the BAL material are the most probable cause for such coordinated variability.

Grier et al. (2015) and Wildy et al. (2015) reach similar conclusions based on the highly variable BAL lines seen in a spectroscopic monitoring campaigns. With variability seen on timescales of just a few days, both authors conclude that the most likely cause of such rapid changes is the BAL gas responding to changes in the incident ionizing continuum.

Leading to an alternative explanation of BAL variability, Capellupo et al. $(2011,2012)$ report on an ongoing monitoring 


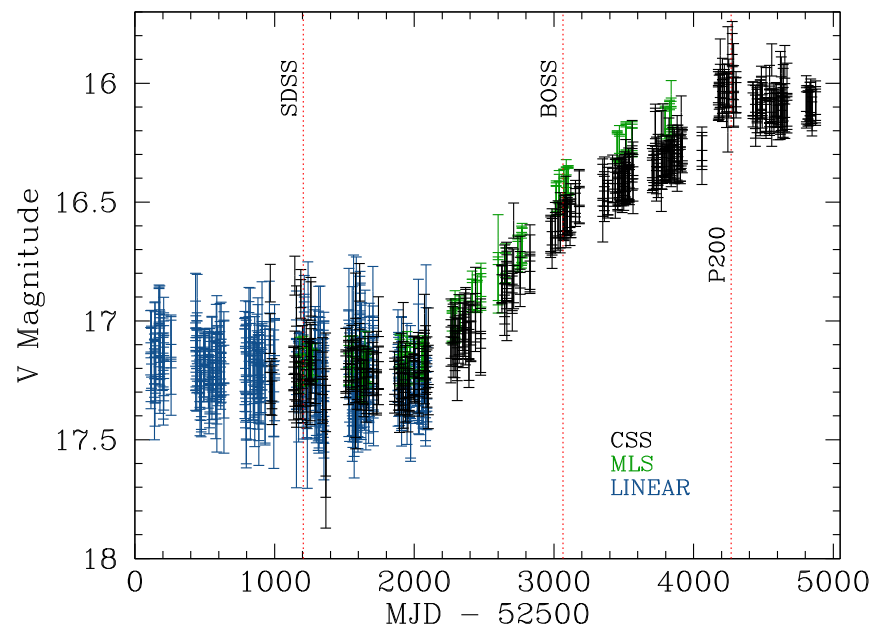

Figure 1. $V$-band light curve for CRTS J0841+2005. CSS and MLS refer to data from two of the telescopes that comprise CRTS, the Catalina Sky Survey $0.7 \mathrm{~m}$ Schmidt telescope located at Mt. Bigelow in Arizona (CSS) and the $1.5 \mathrm{~m}$ Mt. Lemmon Survey Cassegrain reflector, also in Arizona (MLS). LINEAR refers to data from the Lincoln Near-Earth Asteroid Research program, which used two essentially identical $1.0 \mathrm{~m}$ telescopes located at White Sands, New Mexico (Sesar et al. 2011). To put all data on the same photometric scale, offsets have been derived from regions of temporal overlap to ensure equal medians. Vertical lines show the epochs of the optical spectra discussed in Section 2.2.

campaign of a sample of 24 BAL quasars at $1.2<z<2.9$ on timescales ranging from $\sim 4$ months to $\sim 8$ years. Studying the C IV BAL feature, Capellupo et al. (2011) found variability in $40 \%$ of their sample on month-long timescales, and in $65 \%$ of their sample on year-long timescales. They find that highervelocity BALs are more likely to vary than lower-velocity BALs, and that weaker BALs are more likely to vary than stronger BALs. They suggest that the observations are best understood as the movement of clouds within $6 \mathrm{pc}$ of the central engine across the line of sight. In a detailed study of the first observation of $\mathrm{PV} \lambda \lambda 1118,1128 \mathrm{BAL}$ variability in a quasar, Capellupo et al. (2014) argue that the observations are best described by a BAL cloud at a distance of $\lesssim 3.5$ pc moving across the line sight. The implied kinetic energy of the outflow would be $\sim 2 \%$ of the quasar bolometric luminosity, which is sufficient to cause substantial feedback.

Also supporting this interpretation that BAL variability is not dominated by photoionization, He et al. (2014) report on 18 epochs of SDSS/BOSS spectroscopy of a BAL quasar at $z=2.72$. They find only a weak correlation between the BAL variability and the continuum luminosity, suggesting that continuum changes are not driving changes in the BAL trough amplitudes.

Here, we report on CRTS J084133.15+200525.8 (CRTS J0841+2005), an optically bright quasar that has shown extreme variability over the past decade (Figure 1). The quasar transitioned from having a relatively stable visual magnitude of $V \sim 17.3$ between 2002 and 2008, to slowly brightening by a factor of $\sim 2.5$ over the course of 5 years and then plateauing at $V \sim 16.2$. As detailed below, a combination of archival and newly acquired spectroscopy reveal this source to be an iron low-ionization broad absorption line (FeLoBAL) quasar exhibiting extreme spectroscopic changes over the same time period, and the nature of these variations allow us to assess the likely cause of the BAL trough variability.
Independent of our own work on CRTS J0841+2005, Rafiee et al. (2016) recently reported on this same source as part of a sample of three FeLoBAL quasars that have shown significant spectroscopic variability over the past decade. Interestingly, all three show decreasing strength of their low-ionization iron absorption. The current paper has several additions relative to that work. Specifically, we provide new data on CRTS J0841 +2005 , including a new epoch of optical spectroscopy which demonstrates continued spectral changes, and a near-infrared spectrum which provides the first precise redshift for the quasar as well as an estimate of its black hole mass. Finally, Rafiee et al. (2016) remain agnostic as to whether absorber transverse motion or ionization variability is the more likely cause of the changes in the absorption troughs of this source. In contrast, the additional epoch of Palomar spectroscopy presented here allows us to argue that ionization variability is the more likely cause of the extreme absorption variability seen in CRTS J0841+2005.

Throughout this paper, we use Vega magnitudes unless otherwise indicated and we adopt the concordance cosmology, $\Omega_{\mathrm{M}}=0.3, \Omega_{\Lambda}=0.7$ and $H_{0}=70 \mathrm{~km} \mathrm{~s}^{-1} \mathrm{Mpc}^{-1}$.

\section{Data and Results}

\subsection{Optical Light Curve}

The Catalina Real-time Transient Survey ${ }^{6}$ (CRTS; Drake et al. 2009) leverages the Catalina Sky Survey, designed to search for near-Earth objects, as a probe of the time-variable universe. CRTS has used three telescopes for much of the past decade, two in the northern hemisphere and one in Australia, to cover up to $\sim 2500 \mathrm{deg}^{2}$ per night. The filterless observations are broadly calibrated to Johnson $V$ (for details, see Drake et al. 2013) with a nominal depth of $V \sim 20$. The full CRTS data set contains time series for approximately 500 million sources. $^{7}$

CRTS represents the best data set currently available with which to systematically study quasar variability with large samples over a decade-length timescale. In an analysis of characteristic timescales of 240,000 known spectroscopically confirmed objects using Slepian wavelet variance, M. J. Graham et al. (2017, in preparation) originally identified CRTS J0841 +2005 as an extreme outlier in the plane defined by a linear trend (the Thiel-Sen statistic) and deviation from the median Slepian wavelet variance fit. In that analysis, CRTS J0841 +2005 has a characteristic timescale $\tau=109.9$ days, which is significantly larger than expected for a quasar of its magnitude, $\tau=48.0 \pm 5.9$ days.

If we instead characterize quasar light curves with a Gaussian process damped random walk model and only consider the subset of 79,749 quasars with at least 200 CRTS photometric measurements, CRTS J0841+2005 again stands out. The two parameters from this model are the amplitude, $\sigma$, and the characteristic timescale, $\tau$, of the damped random walk (e.g., Kelly et al. 2009). We use a kernel density estimator to determine the distribution of sources in the $\sigma-\tau$ plane, and we find that CRTS J0841+2005 resides in an extreme location in this plane $(\log \Sigma=-7.8$, where $\Sigma$ is the density of sources in this plane). Only seven quasars stand out at this level or more from the population distribution, implying that only $\sim 1$ in 10,000 quasars show variability behavior as extreme as

\footnotetext{
6 See crts.caltech.edu/.

7 See http://catalinadata.org/.
} 

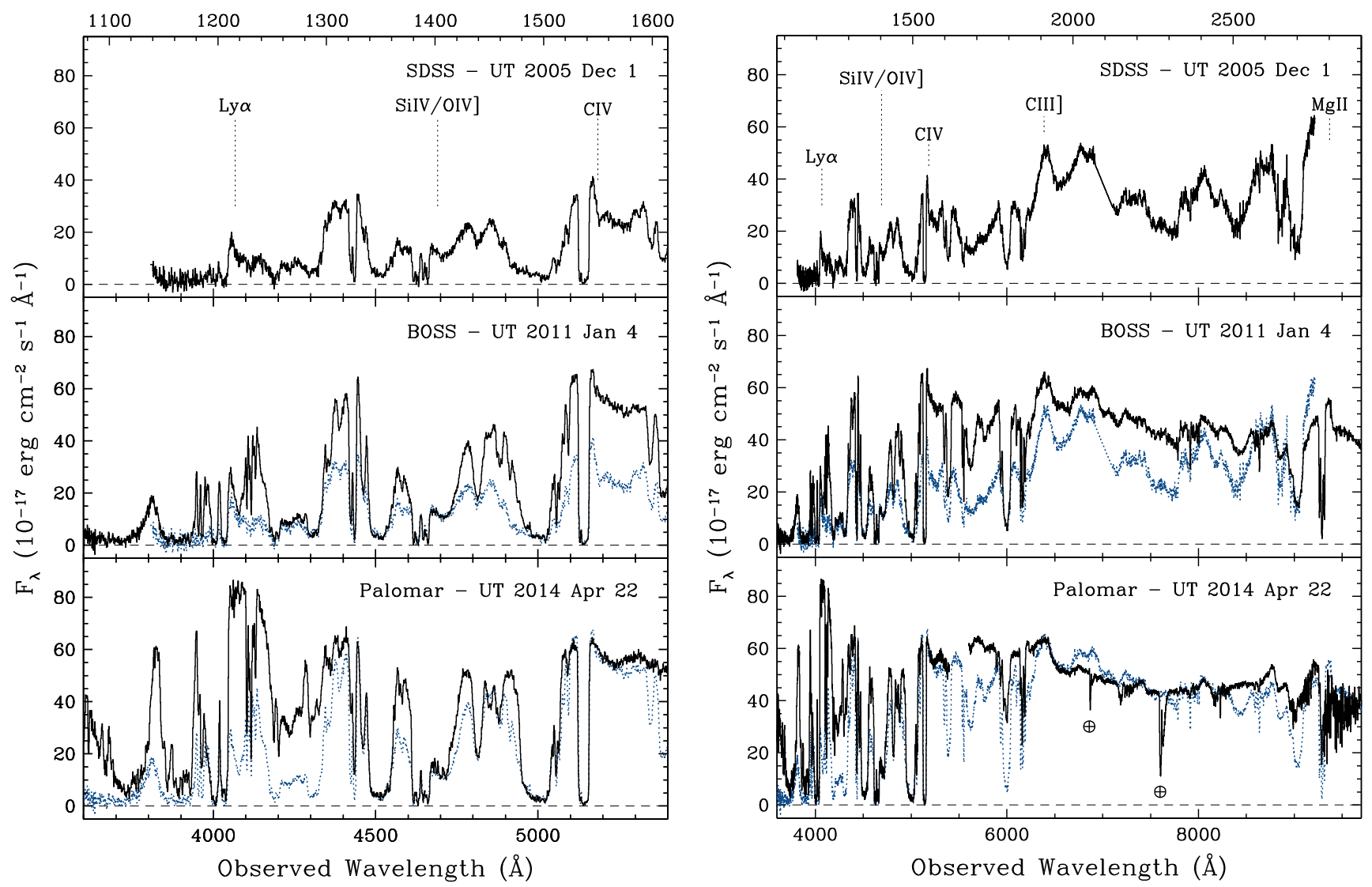

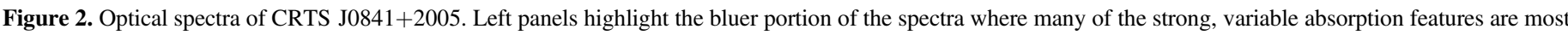

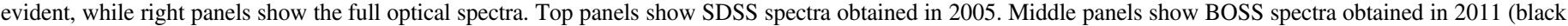

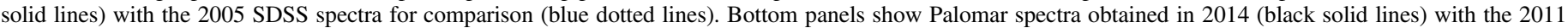

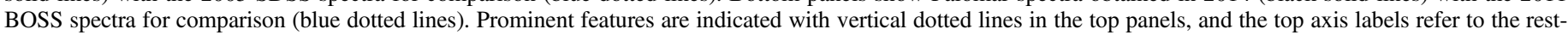
frame wavelength scale, while the bottom axes refer to the observed frame.

CRTS J0841+2005. Further inspection of the CRTS light curve of CRTS J0841+2005 (Figure 1) also indicates that the variable behavior is different from the expected stochastic damped random walk model that describes most quasars, and instead appears more consistent with a state change. Further support for this interpretation comes from earlier photometry of CRTS J0841+2005 reported in Rafiee et al. (2016) from the Palomar Sky Surveys (POSS-I, Palomar Quick V, and POSSII), reaching back to the mid-1950s. Rafiee et al. (2016) reports no evidence for a significant change in the optical brightness of CRTS J0841+2005 prior to 2000.

SDSS imaged CRTS J0841+2005 on UT 2004 December 12 (MJD = 53351), which is prior to the brightening episode. The source was unresolved, and based on its unusual and red colors, SDSS targeted CRTS J0841+2005 for spectroscopic observations as a high-redshift quasar candidate.

\subsection{Optical Spectroscopy}

CRTS J0841+2005 was first observed spectroscopically by SDSS on UT 2005 December 1 (MJD = 53705; Blanton et al. 2003) and was then re-observed by SDSS-III BOSS on UT 2011 January 4 (MJD = 55565; Dawson et al. 2013). The spectra, shown in Figure 2, show a source with many absorption features, making redshift identification challenging. Indeed, the SDSS data releases have reported a variety of redshifts for CRTS J0841+2005, always with warning flags, ranging from $z=0.859$ (DR8; Warning = Many Outliers) to $z=1.295 \quad(\mathrm{DR} 7 ; \quad \mathrm{zStatus}=$ Failed) to $z=3.195 \quad$ (DR9; Warning = Negative Emission). Our visual inspection of the BOSS spectrum tentatively identified $\mathrm{Mg}$ II and Fe II blends in the region around $9400 \AA$, implying $z \sim 2.3$, consistent with both the visual inspection value of $z=2.342$ in the SDSS DR12 quasar catalog (DR12Q; Pâris et al. 2014) and the results of our Keck infrared spectrum described in Section 2.4.

We obtained additional optical spectroscopy of CRTS J0841 +2005 using the Double Spectrograph on the Hale 200" Telescope at Palomar Observatory on UT 2014 April 22 $(\mathrm{MJD}=56769)$. We obtained two $600 \mathrm{~s}$ exposures using the 1 "! 0 slit in cloudy conditions. The data were reduced using standard procedures and relative spectrophotometric calibration was achieved using observations of standard stars obtained on the same night. Figure 2 presents the Palomar data, where we have scaled the spectra so that the long wavelength ( part of the spectra is of comparable flux density to the BOSS spectrum at the same wavelengths.

The multi-epoch spectra show the extreme variability exhibited by CRTS J0841+2005, as well as multiple strong absorption features, characteristic of an FeLoBAL quasar. FeLoBALs are notoriously challenging targets for redshift identification (e.g., Becker et al. 1997; Brunner et al. 2003). We see dramatic changes across the full spectrum, particularly in the spectral region between redshifted $\operatorname{Ly} \alpha$ and C IV. Some features do not change across the near-decade timescale of the 
spectroscopy, such as the saturated C IV absorption at $5150 \AA$. Other features completely disappear, such as absorption lines at $\approx 5450$ and $8500 \mathrm{~A}$. There is an overall uncovering of blue continuum emission, with the flux around Ly $\alpha$ increasing by an order of magnitude over the $>8$ years spanned by the spectroscopy. In addition, while the continuum between $\mathrm{C}$ III] and $\mathrm{Mg}$ II is extremely choppy in the 2005 spectrum, by 2014 it is smoother, which is more typical of normal quasar spectra.

\subsection{Imaging at Other Wavelengths}

CRTS J0841+2005 is a bright near- to mid-infrared source, well detected by both the Two Micron All Sky Survey $\left(K_{s}=13.62 \pm 0.04-2 \mathrm{MASS}\right.$; Skrutskie et al. 2006) and the Wide-field Infrared Survey Explorer $(W 3=9.58 \pm 0.06-$ WISE; Wright et al. 2010). With $W 1-W 2=0.65$, CRTS J0841+2005 is slightly bluer than the mid-infrared AGN selection criteria of Stern et al. (2012), which are $W 1-W 2 \geqslant 0.8$ and $W 2 \leqslant 15.05$. However, as shown in Assef et al. (2013), the AGN selection color can be relaxed for brighter sources.

There is little variability detected at longer wavelengths in this source. In AB magnitudes, the $z$-band magnitude recorded by SDSS was $z=16.37 \pm 0.01$ on MJD 53351, closely matching the $z$-band magnitude of $Z=16.34 \pm 0.01$ recorded by UKIRT Infrared Deep Sky Survey (UKIDSS; Lawrence et al. 2007) on MJD 55141. In the near-infrared (in Vega magnitudes), 2MASS recorded $H=14.41 \pm 0.05$ and $K_{s}=13.62 \pm 0.04$ on MJD 51105, closely matching the UKIDSS values of $H=14.32 \pm 0.02$ on MJD 54061 and $K=13.57 \pm 0.02$ on both MJD 54061 and MJD 55238, where we have assumed a $2 \%$ floor on the UKIDSS photometric calibration (e.g., Hodgkin et al. 2009). Similarly, the mid-infrared flux measured by WISE and NEOWISE (Mainzer et al. 2014) varies by only $\sim 0.04 \mathrm{mag}$, comparable to the typical uncertainty.

CRTS J0841+2005 is not detected by ROSAT, nor was it (serendipitously) observed by either the Chandra X-Ray Observatory or XMM-Newton. CRTS J0841+2005 is also not detected by the Faint Images of the Radio Sky at Twenty $\mathrm{cm}$ survey (FIRST; Becker et al. 1995), implying $S_{1.4 \mathrm{GHz}} \lesssim 1 \mathrm{mJy}$ $(5 \sigma)$. Finally, as expected, observations by the Galaxy Evolution Explorer (GALEX; Martin et al. 2005), which sample below the Lyman limit for $z=2.35$, do not detect CRTS J0841+2005.

\subsection{Near-infrared Spectroscopy}

We obtained a $K$-band (1.95-2.39 $\mu \mathrm{m})$ spectrum of CRTS J0841+2005 with the Multi-Object Spectrometer for InfraRed Exploration (MOSFIRE; McLean et al. 2012; Steidel et al. 2014) on UT 2014 May 5 (MJD = 56782) in longslit mode. We obtained three dithered exposures of $180 \mathrm{~s}$ each through a 0.7 entrance slit under clear conditions with good seeing. The spectrum was reduced using a combination of the MOSFIRE data reduction pipeline (DRP) and custom routines (for details, see Steidel et al. 2014). Wavelength calibration was based on a combination of $\mathrm{OH}$ emission lines in the night sky and an internal $\mathrm{Ne}$ arc lamp. Flux calibration and telluric absorption removal was accomplished using spectra of an A0V star (Vega analog) observed at similar airmass. The final extracted spectrum (Figure 3 ) shows strong continuum and a single broad emission line with a peak at $2.1956 \mu \mathrm{m}$, which we

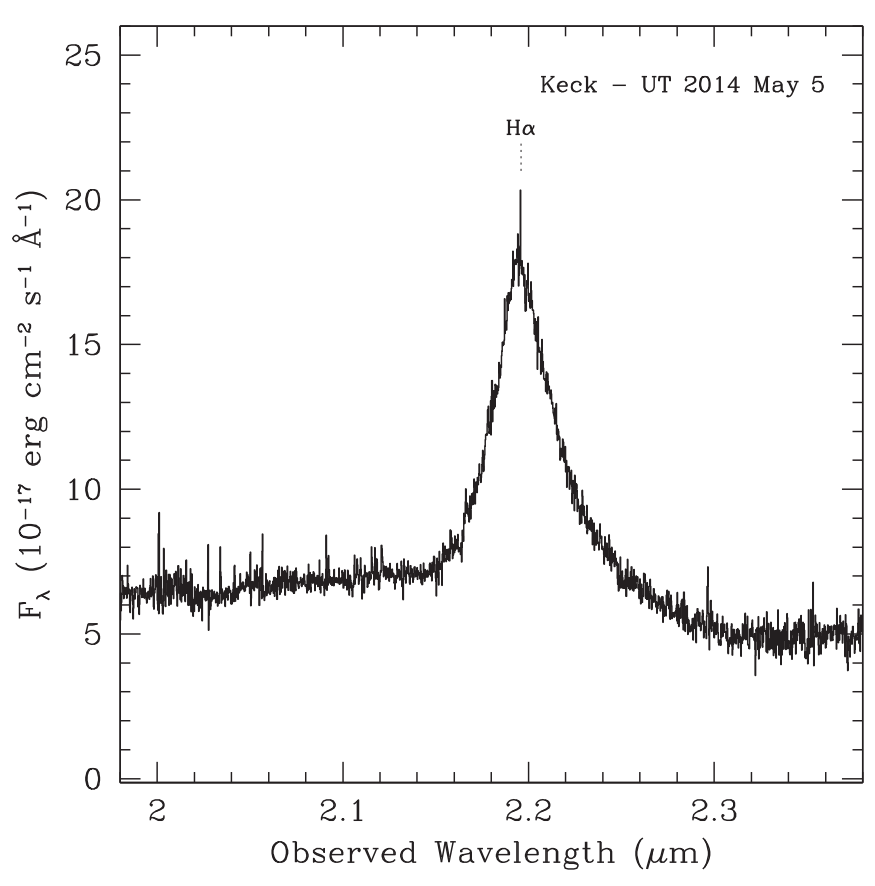

Figure 3. Near-infrared spectrum of CRTS J0841+2005 obtained with Keck/ MOSFIRE. A single, strong, broad emission line is detected, associated with $\mathrm{H} \alpha$.

identify as $\mathrm{H} \alpha$ at $z=2.3446$. The apparent asymmetry in the continuum straddling the line is well modeled by the Boroson $\&$ Green (1992) Fe II template on the blue side of the line.

We use the broad $\mathrm{H} \alpha$ emission line to estimate the mass of the black hole in CRTS J0841+2005. First, we apply a multiplicative correction to the $K$-band spectrum to match the $K$-band photometry from the UKIDSS observations. We then approximate the uncertainties in the spectrum by considering the standard deviation of the spectrum outside the strong emission line. We model the $\mathrm{H} \alpha$ spectral region as the sum of two broad Gaussian lines, a single narrow Gaussian, an iron template (which elevates the continuum on the blue side of the emission line), and a power-law continuum. The full-width at half-maximum (FWHM) of the broad $\mathrm{H} \alpha$ emission is $6086 \pm 42 \mathrm{~km} \mathrm{~s}^{-1}$, and the combined luminosity of the broad $\mathrm{H} \alpha$ components is $L_{\mathrm{H} \alpha}=(5.56 \pm 0.05) \times 10^{45} \mathrm{erg} \mathrm{s}^{-1}$. Modeling the broad-band ( $3000 \AA$ to $7 \mu \mathrm{m}$ ) spectral energy distribution of the quasar as a sum of a power-law continuum, two blackbody thermal components $(500$ and $1250 \mathrm{~K}$, to model the rest-frame IR emission), and line emission from $\mathrm{H} \alpha$ and Fe II as determined from the Keck spectrum, we derive $L_{5100}=(1.24 \pm 0.02) \times 10^{47} \mathrm{erg} \mathrm{s}^{-1}$. Following Jun et al. (2015a), we derive $\log \left(M_{\mathrm{BH}} / M_{\odot}\right)=10.36 \pm 0.16$ using the $L_{5100}$ estimator and $\log \left(M_{\mathrm{BH}} / M_{\odot}\right)=10.29 \pm 0.17$ using the $L_{\mathrm{H} \alpha}$ estimator. We note that these statistical error bars underestimate the true uncertainty, both due to the nonsimultaneity of the imaging and near-infrared spectroscopy and, more importantly, the systematic uncertainty in the virial scale factor, $f$, which is the typically the dominant source of uncertainty in black hole mass measurements; in this case, we adopt $f=5.1 \pm 1.3$ from Woo et al. (2013), as per Jun et al. (2015a).

For comparison, without access to any well-detected emission features, Rafiee et al. (2016) simply adopted a black hole mass of $M_{\mathrm{BH}}=6 \times 10^{9} M_{\odot}$ as a typical value. Adopting 

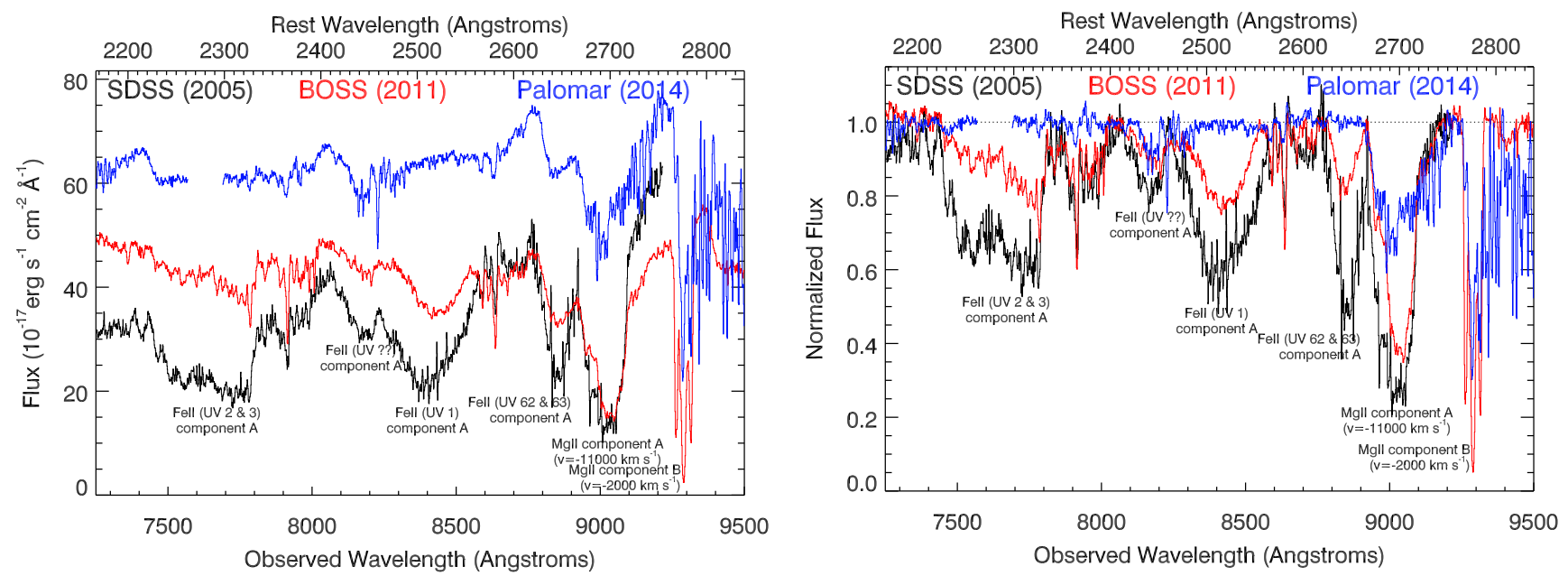

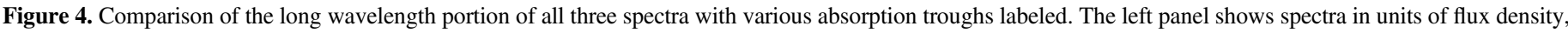

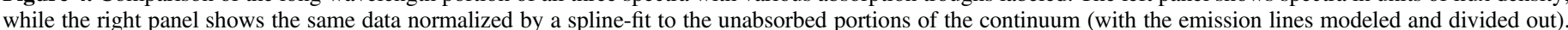

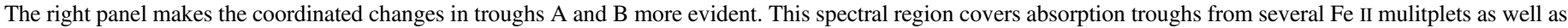
the Mg II doublet. The depth of all absorption troughs became shallower as the overall flux level increased with time.

their value for the bolometric luminosity of CRTS J0841 $+2005, \quad L_{\mathrm{bol}}=(3.36 \pm 0.69) \times 10^{47} \mathrm{erg} \mathrm{s}^{-1} \quad$ (based on the observed rest-frame $2900 \AA$ flux density and a bolometric correction of $\mathrm{BC}_{2900}=5 \pm 1$ from Richards et al. 2006), we determine an Eddington ratio of $L_{\mathrm{bol}} / L_{\mathrm{Edd}} \sim 0.15$ (in comparison to their value of 0.45 ).

\section{Discussion}

Figure 4 shows a comparison between the long wavelength portion of the three epochs of optical spectroscopy. In the spectral region beyond $\sim 7300 \AA$ there is much less blending of troughs from different ions, making the variability changes simpler to interpret. We identify two primary absorption systems. The first system, A, shows several troughs of Fe II UV absorption between 7500 and $8900 \AA$, as well as $\mathrm{Mg}$ II absorption at $9050 \AA$. The other system, B, shows $\mathrm{Mg}$ II absorption at $9300 \AA$. The two absorption systems are separated by $9000 \mathrm{~km} \mathrm{~s}^{-1}$, yet show coordinated reductions in the depth of their troughs as the quasar brightens. This is the expected behavior if the BAL spectral variability is driven by changes in the photoionization: as the ionizing continuum flux increases, the column densities of Fe II and Mg II decrease for all clouds along the line of sight. (Note that this expectation assumes that the ionizing continuum changes are correlated with the flux changes around $2500 \AA$ ). The scenario of clouds moving across our line of sight is hard pressed to explain both the coordinated changes of the trough depths as well as the observed trough weakening with increasing UV flux. A priori, there is no reason that troughs as widely separated in velocity as A and B would be correlated since they are different parcels of gas. Even more so, there is no reason in this scenario for changes in the trough depths to be correlated with flux changes. Therefore, we interpret the variability in the absorption troughs to be due to changes in photoionization, rather than motion of material into our line of sight. A follow-up paper will more carefully model the full multi-epoch spectroscopic data set, including additional spectroscopy from our continuing monitoring, with the goal of understanding the location and energetics of the outflow, and its impact on the host galaxy (C. Chamberlain et al. 2017, in preparation).
CRTS J0841+2005 appears to be an FeLoBAL quasar in the process of transitioning to a more common low-ionization BAL (LoBAL) quasar, similar to FBQS J1408+3054 reported by Hall et al. (2011). We note, however, that Hall et al. (2011) interpreted the variability in that source as being related to structure in the BAL outflow moving out of our line of sight rather than being related to photoionzation changes.

CRTS J0841+2005 highlights the sort of rare, extremely variable quasars that can be used to probe the physics of quasar outflows. We expect to find many more such examples with the new generation of wide-area, sensitive, high-cadence synoptic surveys. We were fortuitous in this case that multi-epoch archival spectroscopy was available for this source. In the future, it will be exciting to find similar major events in real time, allowing real-time multi-wavelength follow-up in order to more fully dissect the internal workings of AGN engines.

We thank the anonymous referee for a prompt and helpful referee report. CRTS was supported by the NSF grants AST1313422, AST-1413600, and AST-1518308. The work of D.S. and H.J. was carried out at Jet Propulsion Laboratory, California Institute of Technology, under a contract with NASA. D.S. also acknowledges support from NASA through ADAP award 12-ADAP12-0109. N.A. and C.C. acknowledge support from NSF through grant AST 1413319, and from NASA through STScI grants GO 11686 and GO 12022. Research by A.J.B. was supported by NSF grant AST1412693. E.G. acknowledges the generous support of the Cottrell College Award through the Research Corporation for Science Advancement. H.J. is supported by an appointment to the NASA Postdoctoral Program at the Jet Propulsion Laboratory, administered by Universities Space Research Association under contract with NASA. The authors are grateful to the staff at the Palomar and Keck observatories, where some of the data presented here were obtained. The authors recognize and acknowledge the very significant cultural role and reverence that the summit of Maunakea has always had within the indigenous Hawaiian community. We are most fortunate to have the opportunity to conduct observations from this mountain. 
Facilities: CRTS, Keck (MOSFIRE), NEOWISE, Palomar (DBSP), SDSS, WISE.

\section{References}

Assef, R. J., Stern, D., Kochanek, C. S., et al. 2013, ApJ, 772, 26 Barlow, T. A., Junkkarinen, V. T., Burbidge, E. M., et al. 1992, ApJ, 397, 81 Becker, R. H., Gregg, M. D., Hook, I. M., et al. 1997, ApJL, 479, 93 Becker, R. H., White, R. L., \& Helfand, D. J. 1995, ApJ, 450, 559 Bentz, M. C., Walsh, J. L., Barth, A. J., et al. 2009, ApJ, 705, 199 Blandford, R. D., \& McKee, C. F. 1982, ApJ, 255, 419

Blanton, M. R., Lin, H., Lupton, R. H., et al. 2003, AJ, 125, 2276

Boroson, T. A., \& Green, R. F. 1992, ApJS, 80, 109

Brunner, R. J., Hall, P. B., Djorgovski, S. G., et al. 2003, AJ, 126, 53

Capellupo, D. M., Hamann, F., \& Barlow, T. A. 2014, MNRAS, 444, 1893

Capellupo, D. M., Hamann, F., Shields, J. C., Halpern, J. P., \& Barlow, T. A. 2012, MNRAS, 422, 3249

Capellupo, D. M., Hamann, F., Shields, J. C., Halpern, J. P., \& Barlow, T. A. 2013, MNRAS, 429, 1872

Capellupo, D. M., Hamann, F., Shields, J. C., Rodríguez Hidalgo, P., \& Barlow, T. A. 2011, MNRAS, 413, 908

Dawson, K. S., Schlegel, D. J., Ahn, C. P., et al. 2013, AJ, 145, 10

D’Orazio, D. J., Haiman, Z., Duffell, P., Farris, B. D., \& MacFadyen, A. I. 2015a, MNRAS, 452, 2540

D’Orazio, D. J., Haiman, Z., \& Schiminovich, D. 2015b, Natur, 525, 351

Drake, A. J., Catelan, M., Djorgovski, S. G., et al. 2013, ApJ, 763, 32

Drake, A. J., Djorgovski, S. G., Mahabal, A., et al. 2009, ApJ, 696, 870

Drake, A. J., Graham, M. J., Djorgovski, S. G., et al. 2011, ApJS, 213, 9

Filiz, Ak, N., Brandt, W. N., Hall, P. B., et al. 2012, ApJ, 757, 114

Filiz, Ak, N., Brandt, W. N., Hall, P. B., et al. 2013, ApJ, 777, 168

Filiz, Ak, N., Brandt, W. N., Hall, P. B., et al. 2014, ApJ, 791, 88

Gezari, S., Hung, T., Cenko, S. B., et al. 2017, ApJ, 835, 144

Gibson, R. R., Brandt, W. N., Gallagher, S. C., Hewett, P. C., \& Schneider, D. P. 2010, ApJ, 713, 220

Gibson, R. R., Brandt, W. N., Schneider, D. P., \& Gallagher, S. C. 2008, ApJ, 675,985

Graham, M. J., Djorgovski, S. G., Drake, A. J., et al. 2014, MNRAS, 439, 703

Graham, M. J., Djorgovski, S. G., Stern, D., et al. 2015a, Natur, 518, 74
Graham, M. J., Djorgovski, S. G., Stern, D., et al. 2015b, MNRAS, 453, 1562 Grier, C. J., Hall, P. B., Brandt, W. N., et al. 2015, ApJ, 806, 111

Hall, P. B., Anosov, K., White, R. L., et al. 2011, MNRAS, 411, 2653

He, Z., Bian, W., Ge, X., \& Jiang, X. 2014, MNRAS, 443, 2532

He, Z., Bian, W., Ge, X., \& Jiang, X. 2015, MNRAS, 454, 3962

Hodgkin, S. T., Irwin, M. J., Hewett, P. C., \& Warren, S. J. 2009, MNRAS, 394, 675

Joshi, R., Chand, H., Srianand, R., \& Majumdar, J. 2014, MNRAS, 442, 862 Jun, H. D., Im, M., Lee, H. M., et al. 2015a, ApJ, 806, 109

Jun, H. D., Stern, D., Graham, M. J., et al. 2015b, ApJL, 814, L12

Kelly, B. C., Bechtold, J., \& Siemiginowska, A. 2009, ApJ, 698, 895

LaMassa, S. M., Cales, S., Moran, E. C., et al. 2015, ApJ, 800, 144

Lawrence, A., Bruce, A. G., MacLeod, C., et al. 2016, MNRAS, 463, 296

Lawrence, A., Warren, S. J., Almaini, O., et al. 2007, MNRAS, 379, 1599

Liu, T., Gezari, S., Heinis, S., et al. 2015, ApJ, 803, 16

Lundgren, B. F., Wilhite, B. C., Brunner, R. J., et al. 2007, ApJ, 656, 73

Macleod, C. L., Ivezić, Ž, Sesar, B., et al. 2012, ApJ, 753, 106

Mainzer, A., Bauer, J., Grav, T., et al. 2014, ApJ, 784, 110

Martin, D. C., Schiminovick, D., Barlow, T. A., et al. 2005, ApJL, 619, L1

Matthews, T. A., \& Sandage, A. R. 1963, ApJ, 138, 30

McGraw, S. M., Shields, J. C., Hamann, F. W., et al. 2015, MNRAS, 453, 1379

McLean, I. S., Steidel, C. C., Epps, H., et al. 2012, Proc. SPIE, 8446, 84460J Pâris, I., Petitjean, P., Aubourg, E., et al. 2014, A\&A, 563, 54

Rafiee, A., Pirkola, P., Hall, P. B., et al. 2016, MNRAS, 459, 2472

Richards, G. T., Lacy, M., Storrie-Lombardi, L. J., et al. 2006, ApJS, 166, 470

Risaliti, G., Elvis, M., \& Nicastro, F. 2002, ApJ, 571, 234

Sesar, B., Scott, S. J., Zeljko, I., et al. 2011, AJ, 142, 190

Skrutskie, M. F., Cutri, R. M., Stiening, R., et al. 2006, AJ, 131, 1400

Steidel, C. C., Rudie, G. C., Strom, A. L., et al. 2014, ApJ, 795, 165

Stern, D., Assef, R. J., Benford, D. J., et al. 2012, ApJ, 753, 30

van den Bergh, S., Herbst, E., \& Pritchet, C. 1973, AJ, 78, 375

Vivek, M., Srianand, R., Petitjean, P., et al. 2012, MNRAS, 423, 2879

Wang, T., Yang, C., Wang, H., \& Ferland, G. 2015, ApJ, 814, 150

Wildy, C., Goad, M. R., \& Allen, J. T. 2014, MNRAS, 437, 1976

Wildy, C., Goad, M. R., \& Allen, J. T. 2015, MNRAS, 448, 2397

Woo, J., Schulze, A., Park, D., et al. 2013, ApJ, 772, 49

Wright, E. L., Eisenhardt, P. R. M., Mainzer, A. K., et al. 2010, AJ, 140, 1868

Zhang, S., Zhou, H., Wang, T., et al. 2015, ApJ, 803, 58 